\title{
Effector-dependent response deterioration by stochastic transformations reveals mixed reference frames for decisions
}

T. Scott Murdison ${ }^{1,2,3}$, Dominic Standage ${ }^{1,2,3}$, Philippe Lefèvre ${ }^{4}$ and Gunnar Blohm ${ }^{1,2,3}$

${ }^{1}$ Centre for Neuroscience Studies, Queen's University, Kingston, Ontario, Canada, K7L3N6

2 Canadian Action and Perception Network (CAPnet), Toronto, Ontario, Canada, M3J1P3

${ }^{3}$ Association for Canadian Neuroinformatics and Computational Neuroscience (CNCN), Kingston, Ontario, Canada, K7L3N6

4 ICTEAM Institute and Institute of Neuroscience (IoNS), Université catholique de Louvain, Louvain-La-Neuve, Belgium, B-1348

39 total pages

6 figures

184 words in Abstract

373 words in Introduction

1415 words in Discussion

0 tables

\section{Conflict of interest statement:}

The authors declare no competing financial interests.

\section{Acknowledgments:}

The authors want to thank the participants for their kind participation in the study and Ashley Clare Parr for her helpful comments on the manuscript. This work was supported by NSERC (Canada), CFI (Canada), the Botterell Fund (Queen's University, Kingston, ON, Canada) and ORF (Canada). TSM was partly supported by DAAD (Germany).

\author{
Abbreviated title: \\ Head roll impairs perceptual decisions \\ Contact: \\ Gunnar Blohm \\ Centre for Neuroscience Studies, Queen's University \\ Botterell Hall, room 229 \\ 18 Stuart St \\ Kingston, Ontario, Canada, K7L 3N6 \\ Email: gunnar.blohm@queensu.ca
}




\section{Abstract}

2 Recent psychophysical and modeling studies have revealed that sensorimotor

3 reference frame transformations (RFTs) add variability to motor output by decreasing

4 the fidelity of sensory signals. How RFT stochasticity affects the sensory input

5 underlying perceptual decisions, if at all, is unknown. To investigate this, we asked

6 participants to perform a simple two-alternative motion direction discrimination task

7 under varying conditions of head roll and/or stimulus rotation while responding either

8 with a saccade or button press, allowing us to attribute behavioral effects to eye-, head-

9 and shoulder-centered reference frames. We observed a rotation-induced, increase in

10 reaction time and decrease in accuracy, indicating a degradation of motion evidence

11 commensurate with a decrease in motion strength. Inter-participant differences in

12 performance were best explained by a continuum of eye-head-shoulder representations

13 of accumulated decision evidence, with eye- and shoulder-centered preferences during

14 saccades and button presses, respectively. We argue that perceptual decision making

15 and stochastic RFTs are inseparable, consistent with electrophysiological recordings in

16 neural areas thought to be encoding sensorimotor signals for perceptual decisions.

17 Furthermore, transformational stochasticity appears to be a generalized phenomenon,

18 applicable throughout the perceptual and motor systems. We show for the first time that,

19 by simply rolling one's head, perceptual decision making is impaired in a way that is

20 captured by stochastic RFTs. 


\section{Significance statement}

22 When exploring our environment, we typically maintain upright head orientations, often

23 even despite increased energy expenditure. One possible explanation for this

24 apparently suboptimal behavior might come from the finding that sensorimotor

25 transformations, required for generating geometrically-correct behavior, add signal-

26 dependent variability (stochasticity) to perception and action. Here, we explore the

27 functional interaction of stochastic transformations and perceptual decisions by rolling

28 the head and/or stimulus during a motion direction discrimination task. We find that,

29 during visuomotor rotations, perceptual decisions are significantly impaired in both

30 speed and accuracy in a way that is captured by stochastic transformations. Thus, our

31 findings suggest that keeping one's head aligned with gravity is in fact ideal for making

32 perceptual judgments about our environment. 


\section{Introduction}

34 We typically maintain upright head and eye orientations with respect to the horizon

35 (Pozzo et al., 1990; Dunbar et al., 2004, 2008), despite potentially increased energy

36 expenditure. For example, during hunting (Land, 2014), flight (Altshuler et al., 2015) or

37 motorcycle racing it would be more energy efficient to align the head with the inertial

38 vector. Minimizing vertical disparity has been suggested as one reason for this behavior

39 (Misslisch et al., 2001; Schreiber et al., 2001). A potential complementary reason could

40 come from the recent finding that reference frame transformations (RFTs) are stochastic

41 (Alikhanian et al., 2015), as is apparent in both perception (Schlicht and Schrater, 2007;

42 Burns et al., 2011) and motor planning (Sober and Sabes, 2003, 2005; McGuire and

43 Sabes, 2009; Burns and Blohm, 2010). If the encoding of evidence is similarly degraded

44 by stochastic transformations, then maintaining specific head orientations while making

45 visuomotor decisions could be optimal for the signal's preservation, despite requiring

46 energy expenditure.

$47 \quad$ Bounded accumulator models account for a wealth of behavioral data from

48 perceptual decision tasks under the premise that noisy evidence for the alternatives is

49 accumulated until it reaches a criterion bound (Smith and Ratcliff, 2004; Bogacz et al.,

50 2006). Under this framework, stochastic RFTs could influence choice behavior in

51 predictable ways. One possibility is that RFTs can degrade the encoding of evidence by

52 lowering its signal-to-noise ratio. In this case, the behavioral outcome should be

53 commensurate with increasing task difficulty, resulting in increased reaction times (RTs)

54 and decreased accuracy (percent correct).

55 The goal of this study was to determine the influence of stochastic RFTs on 
56 perceptual decision making. To do so, participants were asked to perform a 2AFC

57 motion direction discrimination task either under non-rotated (control) conditions or

58 under several different head roll or rotated stimulus conditions (Figure 1). In a blocked

59 design, they were also instructed to indicate their decision regarding the left or right

60 direction of coherent motion with either a saccade or a button press. Because eye

61 movements are executed in head-centered coordinates and, when the arm is stationary,

62 button presses occur in shoulder-centered coordinates, this paradigm allowed us to

63 perform well-established psychometric and chronometric analyses while also allowing

64 us to test the effects of eye-, head- and shoulder-reference frames on choice behavior.

\section{Materials and methods}

68 Experimental paradigm

69 To test how reference frame transformations affect perceptual decisions, we developed

70 an experimental paradigm with distinct conditions consisting of (1) rotations of the visual

71 stimulus, (2) rotations of the head and (3) changes to the response type (saccade or

72 button press). These conditions allowed us to comprehensively investigate the influence

73 of different reference frame transformations on the decision process based on the

74 coding frame of the motion evidence and transformation of that evidence into a

75 reference frame appropriate for the motor response. These conditions are illustrated in

76 Figure $1 \mathrm{~A}$.

77 We determined participants' baseline decision making performance using a

78 control condition in which participants' heads remained upright $\left(0^{\circ}\right.$ roll) and the axis of 
79 coherent motion remained along the horizontal $\left(0^{\circ}\right)$ screen-centered axis. Thus,

80 comparing our other experimental conditions to this one provided the effects directly

81 resulting from adding new requirements to the transformation (Figure $1 \mathrm{~A}$, first column).

82 For each response type, the rotational conditions were rolling the participants' heads

83 towards a shoulder (about $45^{\circ}$ ), without rotation of the on-screen stimulus (head roll -

84 no stimulus rotation, HR-NS, Figure $1 \mathrm{~A}$, second column); head roll with $45^{\circ}$ rotation of

85 the on-screen stimulus (head roll - stimulus rotation, HR-S, Figure 1A, third column);

$8645^{\circ}$ rotation of only the on-screen stimulus (S, Figure $1 \mathrm{~A}$, fourth column).

87

88 Participants

89 In total, 12 participants (age 20-32 years, 8 male) were recruited for two experiments

90 after informed consent was obtained. Eleven of 12 participants were right-handed and

9111 of 12 participants were naïve as to the purpose of each experiment (main and

92 control). Each experiment had seven participants, and two participants performed both

93 main and control experiments. Participants in the main experiment were between the

94 ages of 22 and 32 years ( 5 male) and all were right-handed. Participants in the control

95 experiment were between the ages of 20 and 26 years ( 4 male) and six of seven were

96 right-handed. All participants had normal or corrected-to-normal vision and did not have

97 any known neurological, oculomotor, or visual disorders. All procedures were approved

98 by the Queen's University Ethics Committee in compliance with the Declaration of

99 Helsinki. 


\section{Apparatus}

103 Participants sat in complete darkness $50 \mathrm{~cm}$ in front of a $36 \mathrm{~cm} \times 27 \mathrm{~cm}$ Dell UltraScan

104 P991 CRT monitor (Dell, Round Rock, TX). Participants' heads rested on a chin rest

105 that allowed for head roll in the frontoparallel plane. With their heads in an upright

106 position on the chin rest, the interocular midpoint was aligned to the frontoparallel

107 fixation position on the screen. The visual stimulus was displayed on the screen $(120 \mathrm{~Hz}$

108 refresh rate) using the ViSaGe Visual Stimulus Generator with VSG Toolbox for Matlab

109 (Cambridge Research Systems, Rochester, UK). Movements of both eyes were

110 recorded at $400 \mathrm{~Hz}$ using a Chronos head-mounted 3D video eye tracker (Chronos

111 Vision, Berlin, Germany) that was stabilized to the head using a bite bar. Head

112 movements were recorded at $400 \mathrm{~Hz}$ using an Optotrak Certus system (Northern

113 Digital, Waterloo, Ontario, Canada) with three infrared diode markers placed on the

114 Chronos helmet. For consistency across camera positions, these helmet markers were

115 calibrated with respect to an external orthonormal axis defined by a set of three

116 orthogonal diodes located either on the wall behind the participant or on the side of the

117 CRT monitor. Screen brightness and contrast settings were adjusted so that participants

118 could not see the edges of the monitor screen in complete darkness, even after $0.5 \mathrm{~h}$

119 dark adaptation.

121 Procedure

122 The visual stimulus consisted of a centered array of white circular dots ( $0.1^{\circ}$ diameter)

123 arranged in a circle $\left(10^{\circ}\right.$ diameter $)$, marking the boundary to which participants were

124 instructed to make saccadic responses. At the center of this boundary there was an 
125 aperture $\left(5^{\circ}\right.$ diameter) inside of which we displayed the random dot motion stimulus.

126 The central stimulus was composed of a white fixation point $\left(0.1^{\circ}\right.$ diameter $)$ positioned

127 at the center, and 200 red dots (each $0.1^{\circ}$ diameter) with constant velocities of $4 \%$ s. On

128 each trial we randomly selected a subset of the dots in motion $(2 \%, 10 \%$ or $20 \%$ of all

129 dots) to move coherently in either the leftward or rightward direction. In the stimulus

130 rotation conditions (HR-S and S), we rotated the on-screen motion axis by either $45^{\circ}$ or

$131-45^{\circ}$. In the HR-S condition, this on-screen rotation of motion was congruent with the

132 direction of head roll, such that the motion axis lay approximately along the interocular

133 axis. In all saccadic trials, participants were instructed to make eye movements towards

134 the on-screen $0^{\circ}$ (rightward motion) or $180^{\circ}$ (leftward motion) directions. Participants

135 were also informed of all block conditions (i.e. head roll, visual stimulus rotation) prior to

136 the start of each block.

137 A sample trial progression is illustrated in Figure 1B. At the start of each trial, a

138 fixation dot appeared in the center of the circular saccade boundary (fixation period, 500

$139 \mathrm{~ms}$ ). This fixation period was followed by the visual motion stimulus, displayed within the

140 aperture in the center of the screen along with the fixation point (1500 ms max).

141 Participants were instructed to maintain fixation until they came to a decision about the

142 direction of the coherent motion, and were asked to do so as quickly and as accurately

143 as possible. Depending on the response condition, they either made a saccade along

144 the screen-centered horizontal (left or right) or pressed a button with either their index

145 (left) or middle (right) finger corresponding to the perceived horizontal component of

146 motion. For saccade response trials, participants were instructed to press any button

147 after making a saccade, ending the trial. For button press trials, the decision also ended 
148 the trial. Participants were not given feedback about whether their response was

149 correct. There was an inter-trial interval of $500 \mathrm{~ms}$ during which the screen was

150 completely black.

151 Each participant performed four sessions, each consisting of seven, 100-trial

152 blocks for a total of 2800 trials. All 14 conditions (left and right head rolls and stimulus

153 rotations included) were counter-balanced across all participants using a reduced Latin

154 squares method with an initially randomized list of all conditions. To counterbalance

155 potential learning and fatigue effects, participants performed each condition twice; once

156 in an initial sequence determined by the Latin squares method (Shao and Wei, 1992)

157 and a second time in the reverse sequence. Using this method, each condition was

158 uniformly distributed across all blocks.

160 Raw signal analysis

161 3D head orientation was computed offline as the difference (using quaternion rotation

162 based on (Leclercq et al., 2013)) between a reference upright position measured at the

163 start of each experimental session and head positions throughout the trials. Participants

164 were instructed to begin the first block of each experimental session with an upright

165 head position before responding to the verbal head roll instruction.

166 The eye-in-head orientation was extracted, calibrated and saccades detected

167 using the same techniques as those used by previous work (Blohm and Lefèvre, 2010;

168 Murdison et al., 2013). Briefly, the eye-in-head orientation was extracted after each

169 session from the saved images of the eyes using Iris software (Chronos Vision). This

170 was done using a 9-point grid of calibration dots (10 max eccentricity) with a central 
171 fixation point, while the head remained upright on the chin rest. Each participant was

172 fitted with a customized bite-bar to stabilize the Chronos helmet to the head. Eye-in-

173 head orientation was low-pass filtered (autoregressive forward-backward filter, cutoff

174 frequency $=50 \mathrm{~Hz}$ ) and differentiated twice (weighted central difference algorithm, width

$175=5 \mathrm{~ms}$ ). Saccades were detected using an acceleration threshold of $500 \% \mathrm{~s}^{2}$, as

176 previously done (Blohm and Lefèvre, 2010; Murdison et al., 2013). We defined the eye

177 movement direction as the circular average of horizontal and vertical eye velocity

178 components over the duration of the saccade. For each trial, the head roll measurement

179 was obtained by taking the average head orientation from the motion stimulus onset 180 until the decision time.

182 Trial selection

183 For the main experiment we recorded a total of 19,600 trials from seven participants

184 (2800 trials per participant from four sessions of seven 100-trial blocks each). Of those

185 trials, we removed those that contained a head movement, blink, optokinetic nystagmus

186 or smooth pursuit movement after motion stimulus onset but prior to the decision.

187 Finally, we removed trials on which participants had reaction latencies smaller than 200

$188 \mathrm{~ms}$, as these trials likely represented decisions made preemptively without the use of

189 the visual motion evidence, due to visuomotor processing delays (Thorpe et al., 1996).

190 From the extracted saccades and button presses we determined trial-to-trial directional

191 choices and computed cumulative RT distributions for each rotational condition. For

192 saccades, left or right decisions were classified as saccades whose average direction

193 (based on the entire movement) within a conservative directional window around the 
194 screen-centered horizontal direction $\left(0^{\circ}\right.$ or $\left.180^{\circ}\right)$ with a width of $+/-75^{\circ}$. Trials with

195 saccades with directions outside these windows were removed from the analysis. Also

196 trials for which the participant failed to respond before the end of the 1500 ms response

197 period were removed from analyses (14\% of all trials). Together, these omitted trials

198 comprised $22 \%$ of all trials, leaving 15,274 valid trials.

200 Behavioral analysis

201 We quantified task performance using three main behavioral parameters capturing both 202 speed and accuracy aspects of task performance. These parameters were RT (time

203 elapsed between motion stimulus onset and response), percent error (number of valid

204 incorrect trials divided by the total valid correct and incorrect trials; conversely, percent 205 correct $=100 \%$-percent error), and reward rate (sum of the number of correct trials 206 divided by the sum of all correct and incorrect reaction times). From these parameters 207 we computed the cumulative RT distributions for correct and incorrect trials, to which we 208 fit a modified version of the linear approach to threshold with ergodic rate (LATER) 209 model (Carpenter and Williams, 1995).

210 Because of the short $1500 \mathrm{~ms}$ response window some RT distributions were 211 truncated, resulting in LATER-estimated RT distributions that were not necessarily 212 representative of the data. To account for this issue we fit both correct and incorrect trial 213 RT distributions simultaneously using estimated percent correct as a free parameter 214 that scaled each distribution relative to the other correct (representing percent correct or 215 (100\%-percent error) at $\mathrm{RT}=\infty)$. We also performed all analyses with the empirical 216 percent correct using just the trials within the 1500 ms window and found results 
217 qualitatively similar to those based on the estimated percent correct. We performed the

218 fits using a constrained nonlinear method that minimized the sum of squared residuals.

219 These LATER model fits to the cumulative RT distributions revealed the estimated

220 median reaction latency with its $\mu$ parameter, the approximate slope of the distribution

221 (representing the variability of the distribution) with its $\sigma$ parameter, and the estimated

222 percent correct, each of which we used in behavioral analyses.

We also fit participant and group-level psychometric curves using the Psignifit

224 Toolbox for Matlab (Wichmann and Hill, 2001; Fründ et al., 2011), and fit chronometric 225 data with a scaled logistic function using a nonlinear least squares method. From the 226 psychometric fits we extracted the 75\% PSEs and computed the just-noticeable

227 difference (JND) based on the difference threshold, which is a function of the slope and 228 the midpoint percentile for 2AFC tasks $\pi(=75 \%)$, described by equations (1) and (2):

$$
J N D=2 \times \text { difference threshold }
$$

233 Reference frame analyses

234 We then performed a reference frame analysis on the observed behavioral effects for 235 each rotation condition. To do this, we first made predictions for these effect sizes 236 proportional to the complexity of the RFT in each reference frame (Figure 5A), then 237 computed R-squared coefficients for changes (relative to the non-rotated condition) in 238 RT, percent correct and reward rate. These predictions represented RFTs ranging from 239 highly complex (large effect size), intermediately complex (intermediate effect 
240 size) or simple (no effect), depending on the angle of coherent motion between input at

241 a given reference frame and the required output, which was left or right for either

242 response type. Because they arose from rotations to retinal input due to ocular torsion

243 during head roll (known as ocular counter-roll), intermediate effect sizes were inversely

244 dependent on one another for eye- and head-centered frames. Instead of choosing

245 arbitrary intermediate effect predictions (e.g. 0.5) we optimized the chosen intermediate

246 predictions for each response effector (eye or hand) and each behavioral parameter

247 (RT, percent error or reward rate). This optimization process chose the intermediate

248 prediction that produced the highest across-participant and across-motion coherence

249 mean R-squared value in our reference frame analysis. For saccades, this optimization

250 yielded eye-centered intermediate predictions of 0.83 (latency), 0.54 (percent error) and

2510.59 (reward rate), corresponding to head-centered predictions of $0.17,0.46$ and 0.41

252 respectively. For button presses, this optimization yielded eye-centered intermediate

253 predictions of 0.55 (latency), 0.55 (percent error) and 0.59 (reward rate), corresponding

254 to head-centered predictions of $0.45,0.45$ and 0.41 respectively.

255

256 Control experiment

257 We conducted a control experiment in order to account for potential confounds in our

258 data. Seven participants performed four sessions, each consisting of six, 100-trial

259 blocks (2400 trials per participant) for a total of 16,800 trials, of which we removed $17 \%$

260 of trials for reasons previously listed for the main experiment (see Trial selection),

261 leaving 13,927 valid trials.

262 First, we wanted to ensure that any effects we observed in the stimulus rotated 
263 condition (S) were due to reference frame transformations and not due to participants

264 only accounting for motion along the screen horizontal, which, in the S condition was

265 decreased by a factor of $\sqrt{2}$. To compensate, we introduced a new condition in which

266 the speed of the stimulus was increased by a factor of $\sqrt{2}$ (final speed of $5.7 \% / \mathrm{s}$ ) while

267 the screen stimulus was rotated, called S-spd, depicted in Figure 6A.

268 Second, we wanted to isolate the variability added to the decision process by the

269 initial sensory estimate of head roll. With this in mind, we introduced a condition only for

270 saccadic responses in which the head, stimulus and saccadic response axis were all

271 rotated congruently, called HR-S-RR, depicted in Figure 6A. Therefore, behavior during

272 this condition could be compared to that during the control condition in order to isolate

273 the variability added by head roll. For completeness we included all of the other

274 conditions in the main experiment, and carried out an identical fully counterbalanced

275 and blocked design.

276 Finally, we wanted to ensure that truncation of the RT distributions did not play a

277 role in our observations during the main experiment. Participants were again given

278 instructions to "decide as quickly and accurately as possible," but we allowed them to

279 take up to $5000 \mathrm{~ms}$ to decide the direction of coherent motion, rather than $1500 \mathrm{~ms}$.

280 Participants rarely took the full time to reach a decision ( $0.1 \%$ of all trials). Importantly,

281 this task change did not result in any qualitative differences from our main experiment

282 findings.

283

284 Statistical analyses

285 We performed several n-way ANOVAs (either with 6 or 10 -factors, including interaction 
286 terms) to account for variance in decision making behavior (across RT, percent error 287 and reward rate) due to coherence level, RFT requirements, participant and motor 288 effector. To correct for statistical sampling error, we also carried out a multiple 289 comparison procedure based on Tukey's honestly significant difference criterion. We 290 used the 95\% confidence intervals estimated using Monte-Carlo simulations (Wichmann 291 and Hill, 2001; Fründ et al., 2011) to compare 75\% PSEs and JNDs across RFT 292 conditions in our psychometric analyses.

\section{Results}

296 We utilized several different rotational conditions to determine the effects of saccade297 related (eye-to-head) and button press-related (eye-to-shoulder) reference frame 298 transformations on the performance of a $2 \mathrm{AFC}$ perceptual decision task. Using these 299 conditions, we systematically induced different reference frame transformation 300 requirements under which we analyzed the effects on speed (RT), accuracy (percent 301 error), and net performance (reward rate). This approach allowed us to determine both if 302 changing the RFT requirements had any effect on the integration of decision evidence 303 and, if so, if these effects revealed anything about the coordinate frame of the neural 304 circuitry underlying these decisions.

306 Head and stimulus rotations induced distinct effects on response times and task 307 performance across conditions

308 We found that head and stimulus rotations induced different effects on RT and accuracy 
309 depending on condition. As shown in Figure 2A for example participant 7, cumulative

310 distributions of RTs showed that, depending on the rotation condition, the estimated

311 median RTs shifted by various amounts relative to the control condition in which the

312 head was upright and the stimulus motion axis was horizontally oriented. We also

313 observed overall increases in RT and decreases in accuracy with task difficulty (20\% to

$31410 \%$ to $2 \%$ motion coherence), with each condition inducing different effect magnitudes.

315 These effects depended on the response type, suggesting a potential role for the

316 transformation required to convert sensory input into the response frame used for

317 decision making.

318 Although participants were instructed specifically to perform saccades along their

319 perceived screen-horizontal axis, the absence of visual landmarks around the border of

320 the stimulus allowed us to examine how inducing new rotational conditions altered eye

321 movement generation. As can be seen in Figure 2B for example participant 1, changing

322 the rotation condition resulted in more variable saccade trajectories compared to the

323 control condition. Combined with the observed condition-dependent changes to RT and

324 accuracy, these findings suggest that visuomotor transformations systematically affect

325 the neural processes underlying decision making.

$327 R T$ and percent correct varied with effector, but there was no speed-accuracy tradeoff

328 Not only did each rotational condition induce RT and accuracy effects relative to control,

329 but those effects depended on response type. Figure 3 illustrates this phenomenon with

330 psychometric and chronometric functions at the group level. For example, psychometric

331 functions (left column) show that behavior qualitatively differed between conditions 
332 depending on whether participants responded with a saccade or button press. Under

333 the HR-NS condition (grey), participants performed similarly to control and reached the

334 overall $75 \%$ correct threshold (or 2AFC psychometric PSE) at the lowest motion

335 coherence of any condition (left inset). The JND, which is defined as the ratio

336 representing the units of motion strength (\% coherence) required to increase the

337 percent correct (\%) by a single unit, reveals that participants also tended to perform with

338 the highest precision in the HR-NS condition (right inset). After the HR-NS condition, the

339 control, HR-S (red) and S (cyan) conditions follow in overall accuracy (PSE) and

340 precision (JND) across motion strengths, with S condition JNDs differing significantly

341 from those of other conditions $(p<0.05)$. Comparing directly to button responses (lower

342 left panel), one can see that this pattern is qualitatively different, with the control

343 condition having the lowest PSE of the conditions $(p<0.05)$, followed by HR-NS, HR-S,

344 and S. The saccadic response chronometric functions (upper right panel), represented

345 across motion coherences and conditions reveal how RT distributions varied with

346 motion coherence and rotational condition. Across all coherence levels, RTs were

347 smallest in the control condition, followed by the nearly identical HR-NS and HR-S

348 conditions, and finally the S condition.

349 Taken together, these observations suggest that there was an overall

350 degradation of the encoded evidence and no clear speed-accuracy tradeoff (SAT)

351 across rotational conditions. Similarly, button press responses showed a pattern of

352 psychometric (lower left panel) and chronometric (lower right panel) changes

353 suggesting an overall degradation of the encoding of evidence by rotational changes

354 rather than an SAT (Standage et al., 2014b). Additionally, the observed effector specific 
355 patterns of performance changes across condition suggest that the reference frame of

356 the motor response played a role in the encoding of evidence.

However, we observed high amounts of inter-participant behavioral variability.

358 We show this variability for changes in RT, percent error and reward rate in Figure 4

359 across all participants (colored line segments on left axes). We also observed some

360 consistent trends across task difficulty $(\mathrm{RT}: F(2)=12.73, p<0.01$; percent error: $F(2)=$

$361326.5, p<0.01$; reward rate: $F(2)=33.54, p<0.01)$, rotation condition $(\mathrm{RT}: F(3)=7.78$,

$362 p<0.01$; percent error: $F(3)=4.76, p<0.05$; reward rate: $F(2)=34.25, p<0.01)$ and

363 effector (reward rate: $F(1)=21.58, p<0.01$ ). Note that for reward rate (bottom row), the

$364 \mathrm{y}$-axes are inverted for visualization purposes. On average (inset bars on right axes),

365 participants had longer RTs and had lower reward rates when making decisions under

366 the S condition (cyan bars), when compared to control (multiple comparison $p<0.05$ ),

367 HR-NS (grey; multiple comparison $p<0.05$ ), and HR-S (red; multiple comparison $p<$

368 0.05) conditions. Importantly, we did not see an SAT, as reward rate also decreased

369 (bottom row) with increases in both RT and percent error. These behavioral changes

370 were, however, consistent with a degradation of evidence encoding such that the task

371 was more difficult under rotated conditions, and that difficulty increased with RFT

372 complexity. We observed participant-specific differences in RT between effectors

373 (interaction effect, $F(6)=4.93, p<0.01$ ) and between RFT condition (interaction effect,

$374 F(18)=3.03, p<0.01)$. For example, one can see differences between saccade and

375 button responses for participant \#5 (blue traces) or for participant \#3 (yellow traces)

376 across each effector and coherence level. This trend suggests that the noise added to

377 the evidence encoding not only changed with effector, but also with rotational condition, 
378 in agreement with the observed changes to psychometric and chronometric functions.

379 We next used a reference frame approach to determine the source of this additive noise

380 in the decision process.

381

382 Reference frame analysis

383 To quantify this inter-participant variability, we interpreted the effects using predictions

384 from stochastic reference frame transformations (Alikhanian et al., 2015). We did this

385 under the assumption that the motion information used in the decision was impaired to

386 an extent that was proportional to the complexity of the required visuomotor rotation.

387 Using this approach, we predicted the size of each effect, relative to control, according

388 to the required rotation for a correct effector-centered response in each condition, which

389 we illustrate in Figure 5A. For example, consider the eye-centered prediction for the

390 condition in which both the head and the screen were rotated and a saccadic response

391 was required (HR-S; middle cell, top row, top grid, panel A): in order to correctly

392 interpret the spatial motion direction using eye-centered information, the brain must

393 rotate the retinal vector (which points along its horizontal; for visualization see Figure

$3941 \mathrm{~A})$ by the head roll magnitude to generate a screen-centered horizontal saccade. This

395 requirement differs for the condition in which the head, but not the stimulus, was rotated

396 (HR-NS). Because the retinal vector was rotated solely by head roll and ocular counter-

397 roll, and the eyes are also rotated along with the head, the brain only needed to account

398 for ocular counter-roll when transforming the retinal vector into a screen-horizontal

399 saccade. Therefore, in the eye-centered case, we predicted a large stochastic effect for

400 HR-S (black shading) due to head roll and an intermediate effect for HR-NS (grey 
401 shading) due to only ocular counter-roll. In this way, we made predictions for each

402 effector and for each reference frame (eye, head and shoulder).

403 Using these predictions, we computed the R-squared coefficients for each

404 behavioral parameter (RT, percent error and reward rate), each participant, each

405 effector and each motion coherence. These are depicted in Figure 4B along with the

406 predictions for a purely eye-centered (red dot), head-centered (blue dot) and shoulder-

407 centered (green dot) codings. Each R-square coefficient is color-coded according to

408 participant and represented by a symbol depending on response type (saccades: open

409 disk, button: filled square). Across both RT and percent error at $20 \%$ coherence, the R-

410 square coefficients suggest that evidence was being encoded according to a continuum

411 of reference frames between eye and shoulder, with a strong head-centered component

412 in some cases (e.g. button press responses of participant 5).

413 The transformation-related effect was also dependent on the strength of the

414 stimulus, indicating that the addition of variability to the encoded evidence depended on

415 the initial strength of visual motion. For example, while there is a clear organization of

416 R-square coefficients for the $20 \%$ and $10 \%$ motion coherence conditions for changes in

417 latency along an eye-head-shoulder continuum (Fig 5B, upper left and middle panels),

418 this continuum becomes less clear when the stimulus strength is decreased at $2 \%$

419 motion coherence (Fig 5B, upper right panel).

420 With this analysis, we quantified the effector specific component that we initially

421 observed in the psychometric and chronometric functions (Figure 3). This component

422 was strongest when considering reward rate (bottom row of Figure 5B). Across motion

423 coherence, group reward rate averages (black symbols) indicated that evidence leading 
424 to saccadic responses was more eye-centered while evidence leading to button

425 responses was more shoulder-centered. This trend suggests that the neural circuitry

426 encoding decision evidence is tied to the motor plan for the upcoming movement.

427 Additionally, this mixture of eye- and shoulder-centered components indicates that there

428 could be some concomitant evidence coding by eye- and shoulder-related areas during

429 integration, regardless of eventual motor effector.

431 Control experiment

432 Our main experiment had two important limitations: (1) in the stimulus-rotated condition

$433 \mathrm{~S}$ we could not definitively rule out the influence of decreased motion energy along the

434 screen horizontal during the integration of motion evidence, and (2) we could not isolate

435 the effects of only head roll on the decision process. To address these limitations, we

436 re-ran the experiment with a new group of participants with two added conditions: (1)

437 screen rotation with a proportional increase in the speed of the stimulus to compensate

438 for the loss of horizontal motion energy in the initial S condition (S-speed, green) and (2)

439 head roll with rotation of the screen stimulus and saccadic responses rotated along the

440 motion axis, and not screen horizontal (HR-S-RR, purple), depicted in Figure 6A.

441 Importantly, this experiment produced similar statistical RT, accuracy and reward

442 rate effects as the main experiment for the repeated RFT conditions across task

443 difficulty, motor effector, rotation condition and participant. Shown in Figure 6B, the

444 cumulative RTs for participant 4 show that both the S-spd and HR-S-RR conditions

445 each produced behaviors similar to their conditional counterparts (note that this is a 446 different participant 4 than in the main experiment). We detected no differences in RT, 
447 percent error or reward rate due to the RFT between S-spd and S or between HR-S-RR

448 and HR-S, but found one significant RFT effect between control and HR-S-RR for only

449 percent error $(F(1)=9.10, p=0.03$, for $\mathrm{RT}$ and reward rate all $p>0.05)$. These findings

450 indicate that (1) there was no detectable behavioral effect of the decrease in horizontal

451 motion energy during the $S$ condition in the main experiment, thus validating our initial

452 findings, and (2) the behavioral effects we observed under head roll conditions resulted

453 from the transformation itself and not from a noisy initial sensory estimate of head roll.

455 Discussion

456 Summary of findings

457 The goal of this study was to determine the influence of stochastic reference frame 458 transformations on decision making. We designed a paradigm in which 7 participants 459 performed a 2AFC motion direction discrimination task under control conditions (head 460 upright, stimulus motion along the screen horizontal) or under one of several rotation 461 conditions in which the head and/or stimulus were rotated. Combining rotation 462 conditions with saccadic and button responses allowed us to behaviorally quantify eye-, 463 head- and shoulder-centered effects.

464 We made predictions for the influence of RFTs on speed (RT), accuracy (percent 465 error/correct) and overall performance (reward rate). We found (1) that stochastic 466 reference frame transformations impair decision making, leading to slower, less 467 accurate decisions, (2) that this stochasticity is added in a manner consistent with a 468 mixed eye-head-shoulder representation of evidence, and (3) that within this continuum 469 there is an effector specific component, with saccadic responses more closely 
470 resembling eye-centered predictions and button responses more closely resembling

471 shoulder-centered predictions. Our findings are consistent with the hypothesis that

472 perceptual decision making and visuomotor reference frame transformations occur

473 within the same neural circuitry (Dorris et al., 1997; Gold and Shadlen, 2000), and as

474 such are consistent with the affordance competition hypothesis of embodied decision

475 making, which predicts that motor planning for perceptual decision making occurs in

476 parallel between networks coding for multiple potential actions (for reviews see Cisek

477 2007; Cisek and Pastor-Bernier 2014).

$478 \quad$ Although both evidence integration and motor preparation are often necessary

479 for choice behavior, it is often difficult to distinguish between the contributions of each

480 using standard perceptual tasks. Previous efforts to do so include using delays between

481 stimulus viewing and motor response (Shadlen and Newsome, 2001; Sommer and

482 Wurtz, 2001; Lemus et al., 2007), limiting stimulus viewing time (Bergen and Julesz,

483 1983; Ratcliff and Rouder, 2000; Bodelón et al., 2007; Kiani et al., 2008) and even

484 "compelling" the movement by informing the perceptual system ahead of time about the

485 target characteristics (Salinas et al., 2014). At the neural level, perceptual and motor

486 processes both occur in sensorimotor association areas (Munoz and Wurtz, 1995;

487 Dorris et al., 1997; Horwitz and Newsome, 1999; Shadlen and Newsome, 2001;

488 Hernández et al., 2010; Costello et al., 2013; Mante et al., 2013). Not only are our

489 findings consistent with these neurophysiological principles, but we have also now

490 quantified this inseparability for the first time within an RFT framework. 
Open questions

494 We found that transformation-induced stochasticity impairs decision making. Given that

495 psychophysical thresholds are systematically lowered by the added RFT noise, the

496 simplest explanation points to a degradation of the encoding of motion evidence most

497 likely in the middle temporal (MT) or medial superior temporal (MST) areas (Albright,

498 1984; Britten et al., 1992, 1993, 1996; Salzman et al., 1992; Inaba et al., 2007). MT and

499 MST are highly interconnected areas that serve as the interface between retinal motion

500 signals and the rest of the visuomotor pathways (Ungerleider and Desimone 1986;

501 Komatsu and Wurtz 1988; Newsome et al. 1988; Ilg and Thier 2003; Inaba et al. 2011;

502 for review see Krauzlis 2004), and exhibit gain modulation and receptive field shifts

503 (Chukoskie and Movshon, 2009; Fujiwara et al., 2011; Inaba et al., 2011)

504 mechanistically consistent with carrying out 3D visuomotor transformations (Blohm and

505 Crawford, 2007; Blohm et al., 2009; Blohm and Lefèvre, 2010; Blohm, 2012; Murdison

506 et al., 2015). If these areas indeed provide the neural substrate for the addition of

507 variability to visual motion signals via RFTs, then gain modulation for RFTs itself could

508 be a stochastic process - a question that should be investigated in future

509 electrophysiological and modeling work.

$510 \quad$ Our findings suggest that the encoding of evidence was shared by effectors.

511 However, the contents of that signal differed between participants - something that

512 might be explained by inter-participant differences in the variability of the evidence

513 integration. RFT stochasticity added to the integration process could result in a less

514 reliable population 'readout' of the current estimate of stimulus motion by downstream

515 areas, resulting in more variable RT distributions with shallower slopes (Carpenter and 
516 Williams, 1995). Although we did not see any clear indications of this on LATER model

517 slopes ( $\sigma$; see Methods), differences in how these population responses are decoded by

518 structures closer to the motor output such as the superior colliculus (SC) (Munoz and

519 Wurtz, 1995; Dorris et al., 1997; Horwitz and Newsome, 1999; Sommer and Wurtz,

5202001 ) or primary motor cortex (M1) (Riehle and Requin, 1989; Crammond and Kalaska,

521 1996, 2000) could potentially explain some of the inter-participant variability we

522 observed in RT, percent error and reward rate correlations.

524 Potential mechanism and underlying neural circuitry

525 Our findings are consistent with the hypothesis that the encoding of motion evidence is

526 degraded by RFTs; however, this was not the only possible way that RFTs could have

527 affected decision making. For example, changes in background noise could have

528 modulated the dynamics of circuitry integrating evidence (Furman and Wang, 2008;

529 Roxin and Ledberg, 2008; Standage et al., 2013, 2014a, 2014b), consistent with recent

530 data (Heitz and Schall, 2012). If so, SAT would have been observed (Standage et al., $5312014 b)$.

532 The finding that the impairment of performance relied partially on the response

533 type implies the existence of two partially distinct perceptual decision making networks

534 between behavioral effectors, as previously theorized (Dean et al., 2011; Madlon-Kay et

535 al., 2013). In the lateral intraparietal area (LIP) and the parietal reach region (PRR),

536 which lies along the medial bank of the intraparietal sulcus (IPS), population-level neural

537 activity has been shown to reflect an effector-nonspecific movement signal until a

538 monkey makes a decision regarding which effector to use, at which point PRR activity is 
539 associated with a reach (Cui and Andersen, 2007; Yttri et al., 2014; Wong et al., 2016)

540 or LIP activity is associated with a saccade (Cui and Andersen, 2007; Wong et al.,

541 2016). To accomplish this, recent electrophysiological findings (Wong et al., 2016)

542 indicate that there are ensembles of neurons on both the medial and lateral banks of the

543 IPS that are active during the decision process. Specifically, Wong and colleagues

544 (2016) found an ensemble of neurons that predict the upcoming decision, independent

545 of effector specific region, that coherently spike prior to effector specific local ensembles

546 in each bank (Wong et al., 2016), consistent with previous findings (Cui and Andersen,

547 2007; Yttri et al., 2014). These partially distinct neural ensembles could therefore give

548 rise to the mixture of reference frames our perceptual findings imply should be present

549 in the neural integration of motion evidence. Of course, this explanation does not

550 preclude perceptual and motor contributions from other effector nonspecific areas such

551 as the prefrontal cortex (Madlon-Kay et al., 2013) or from other effector specific areas

552 whose activities are believed to implement a decision variable such as FEF (Hanes and

553 Schall, 1996; Gold and Shadlen, 2000, 2003; Sommer and Wurtz, 2001) or the dorsal

554 premotor cortex (Crammond and Kalaska, 1996, 2000; Cisek and Kalaska, 2002, 2005),

555 or downstream in SC (Munoz and Wurtz, 1995; Dorris et al., 1997; Sommer and Wurtz,

556 2001; White et al., 2013) or M1 (Riehle and Requin, 1989; Crammond and Kalaska,

557 1996, 2000). The precise role that RFT stochasticity plays within such a distributed

558 perceptual decision network, especially with several anatomically distinct sensorimotor

559 association areas with different information flow characteristics and latencies is unclear

560 (Siegel et al., 2015). Furthermore, within these areas, it is also unclear how local neural

561 population codes vary with body and spatial geometry during visuomotor decisions. 
562 These are questions that should be further investigated psychophysically and 563 electrophysiologically.

564 Our findings have implications for studies involving the integration of evidence for 565 movement, whether used for perceptual decision making or motor preparation. First, we 566 found that RFT stochasticity affects the encoding of evidence for perceptual decision 567 making, bringing to light the requirement for controlling the visuomotor geometry during 568 perceptual tasks. Second, the finding that this added variability was partially effector 569 specific could explain some variability between psychophysical performance when the 570 perceptual task is identical with the exception of the motor response (Palmer et al., 571 2005).

572 The influence of RFT stochasticity on perceptual decision making is consistent 573 with previous findings in visuomotor tasks (Sober and Sabes, 2003, 2005; Schlicht and

574 Schrater, 2007; McGuire and Sabes, 2009; Burns and Blohm, 2010; Burns et al., 2011),

575 suggesting that it represents a generalized phenomenon wherever RFTs can be found 576 throughout the perceptual and motor systems. Whether this phenomenon can be further 577 extended to processes requiring a higher degree of cognitive involvement such as 578 strategic decision making or memory storage and retrieval remain open questions. 


\section{References}

582 Albright TD (1984) Direction and orientation selectivity of neurons in visual area MT of $583 \quad$ the macaque. J Neurophysiol 52:1106-1130.

584 Alikhanian H, Carvalho SR, Blohm G (2015) Quantifying effects of stochasticity in 585 reference frame transformations on posterior distributions. Front Comput Neurosci $586 \quad 9: 1-9$

587 Altshuler DL, Bahlman JW, Dakin R, Gaede AH, Goller B, Lentink D, Segre PS, 588 Skandalis DA (2015) The biophysics of bird flight: functional relationships integrate 589 aerodynamics, morphology, kinematics, muscles, and sensors. Can J Zool 93:961590975.

591 Bergen JR, Julesz B (1983) Parallel versus serial processing in rapid pattern 592 discrimination. Nature 303:696-698.

593 Blohm G (2012) Simulating the cortical 3D visuomotor transformation of reach depth. $594 \quad$ PLoS One 7:e41241.

595 Blohm G, Crawford JD (2007) Computations for geometrically accurate visually guided $596 \quad$ reaching in 3-D space. J Vis 7:1-22.

597 Blohm G, Keith GP, Crawford JD (2009) Decoding the cortical transformations for $598 \quad$ visually guided reaching in 3D space. Cereb cortex 19:1372-1393.

599 Blohm G, Lefèvre P (2010) Visuomotor Velocity Transformations for Smooth Pursuit $600 \quad$ Eye Movements. J Neurophysiol 104:2103-2115.

601 Bodelón C, Fallah M, Reynolds JH (2007) Temporal resolution for the perception of 602 features and conjunctions. J Neurosci 27:725-730.

603 Bogacz R, Brown E, Moehlis J, Holmes P, Cohen JD (2006) The physics of optimal 
604 decision making: a formal analysis of models of performance in two-alternative 605 forced-choice tasks. Psychol Rev 113:700-765.

606 Britten KH, Newsome WT, Shadlen MN, Celebrini S, Movshon JA (1996) A relationship 607 between behavioral choice and the visual responses of neurons in macaque MT. $608 \quad$ Vis Neurosci 13:87-100.

609 Britten KH, Shadlen MN, Newsome WT, Movshon JA (1992) The analysis of visual 610 motion: a comparison of neuronal and psychophysical performance. J Neurosci $611 \quad 12: 4745-4765$.

612 Britten KH, Shadlen MN, Newsome WT, Movshon JA (1993) Responses of neurons in 613 macaque MT to stochastic motion signals. Vis Neurosci 10:1157-1169.

614 Burns JK, Blohm G (2010) Multi-sensory weights depend on contextual noise in 615 reference frame transformations. Front Hum Neurosci 4:1-15.

616 Burns JK, Nashed JY, Blohm G (2011) Head roll influences perceived hand position. J $617 \quad$ Vis 11:1-9.

618 Carpenter $\mathrm{RH}$, Williams ML (1995) Neural computation of log likelihood in control of 619 saccadic eye movements. Nature 377:59-62.

620 Chukoskie L, Movshon JA (2009) Modulation of visual signals in macaque MT and MST 621 neurons during pursuit eye movement. J Neurophysiol 102:3225-3233.

622 Cisek P (2007) Cortical mechanisms of action selection: the affordance competition 623 hypothesis. Philos Trans R Soc Lond B Biol Sci 362:1585-1599.

624 Cisek P, Kalaska JF (2002) Simultaneous encoding of multiple potential reach 625 directions in dorsal premotor cortex. J Neurophysiol 87:1149-1154.

626 Cisek P, Kalaska JF (2005) Neural correlates of reaching decisions in dorsal premotor 
627 cortex: Specification of multiple direction choices and final selection of action. $628 \quad$ Neuron 45:801-814.

629 Cisek P, Pastor-Bernier A (2014) On the challenges and mechanisms of embodied 630 decisions. Philos Trans R Soc London B Biol Sci 369:20130479.

631 Costello MG, Zhu D, Salinas E, Stanford TR (2013) Perceptual modulation of motor--but 632 not visual--responses in the frontal eye field during an urgent-decision task. J $633 \quad$ Neurosci 33:16394-16408.

634 Crammond DJ, Kalaska JF (1996) Differential relation of discharge in primary motor 635 cortex and premotor cortex to movements versus actively maintained postures 636 during a reaching task. Exp Brain Res 108:45-61.

637 Crammond DJ, Kalaska JF (2000) Prior information in motor and premotor cortex: 638 activity during the delay period and effect on pre-movement activity. J Neurophysiol $639 \quad 84: 986-1005$.

640 Cui H, Andersen RA (2007) Posterior Parietal Cortex Encodes Autonomously Selected $641 \quad$ Motor Plans. Neuron 56:552-559.

642 Dean HL, Martí D, Tsui E, Rinzel J, Pesaran B (2011) Reaction Time Correlations 643 during Eye-Hand Coordination:Behavior and Modeling. J Neurosci 31:2399-2412.

644 Dorris MC, Paré M, Munoz DP (1997) Neuronal activity in monkey superior colliculus 645 related to the initiation of saccadic eye movements. J Neurosci 17:8566-8579.

646 Dunbar DC, Badam GL, Hallgrímsson B, Vieilledent S (2004) Stabilization and mobility 647 of the head and trunk in wild monkeys during terrestrial and flat-surface walks and $648 \quad$ gallops. J Exp Biol 207:1027-1042.

649 Dunbar DC, Macpherson JM, Simmons RW, Zarcades A (2008) Stabilization and 
mobility of the head, neck and trunk in horses during overground locomotion: comparisons with humans and other primates. J Exp Biol 211:3889-3907.

652 Fründ I, Haenel NV, Wichmann FA (2011) Inference for psychometric functions in the 653 presence of nonstationary behavior. J Vis 11:1-19.

654 Fujiwara K, Akao T, Kurkin S, Fukushima K (2011) Activity of pursuit-related neurons in 655 medial superior temporal area (MST) during static roll-tilt. Cereb Cortex 21:155656165.

657 Furman M, Wang X-J (2008) Similarity effect and optimal control of multiple-choice 658 decision making. Neuron 60:1153-1168.

659 Gold JI, Shadlen MN (2000) Representation of a perceptual decision in developing 660 oculomotor commands. Nature 404:390-394.

661 Gold JI, Shadlen MN (2003) The influence of behavioral context on the representation 662 of a perceptual decision in developing oculomotor commands. J Neurosci 23:632663651.

664 Hanes DP, Schall JD (1996) Neural control of voluntary movement initiation. Science $665 \quad 274: 427-430$.

666 Heitz RP, Schall JD (2012) Neural Mechanisms of Speed-Accuracy Tradeoff. Neuron $667 \quad 76: 616-628$.

668 Hernández A, Nácher V, Luna R, Zainos A, Lemus L, Alvarez M, Vazquez Y, Camarillo 669 L, Romo R (2010) Decoding a perceptual decision process across cortex. Neuron $670 \quad 66: 300-314$.

671 Horwitz GD, Newsome WT (1999) Separate signals for target selection and movement 672 specification in the superior colliculus. Science 284:1158-1161. 
673 Ilg UJ, Thier P (2003) Visual tracking neurons in primate area MST are activated by 674 smooth-pursuit eye movements of an "imaginary" target. J Neurophysiol 90:1489$675 \quad 1502$.

676 Inaba N, Miura K, Kawano K (2011) Direction and speed tuning to visual motion in 677 cortical areas MT and MSTd during smooth pursuit eye movements. J Neurophysiol $678 \quad 105: 1531-1545$.

679 Inaba N, Shinomoto S, Yamane S, Takemura A, Kawano K (2007) MST neurons code 680 for visual motion in space independent of pursuit eye movements. J Neurophysiol $68197: 3473-3483$.

682 Kiani R, Hanks TD, Shadlen MN (2008) Bounded integration in parietal cortex underlies 683 decisions even when viewing duration is dictated by the environment. J Neurosci $684 \quad 28: 3017-3029$.

685 Komatsu H, Wurtz RH (1988) Relation of cortical areas MT and MST to pursuit eye 686 movements . III . Interaction with full-field visual stimulation. J Neurophysiol $687 \quad 60: 621-644$.

688 Krauzlis RJ (2004) Recasting the smooth pursuit eye movement system. J Neurophysiol $689 \quad 91: 591-603$.

690 Land MF (2014) Eye movements of vertebrates and their relation to eye form and 691 function. J Comp Physiol A Neuroethol Sensory, Neural, Behav Physiol 201:195692214.

693 Leclercq G, Lefèvre P, Blohm G (2013) 3D kinematics using dual quaternions: theory 694 and applications in neuroscience. Front Behav Neurosci 7:1-25.

695 Lemus L, Hernández A, Luna R, Zainos A, Nácher V, Romo R (2007) Neural correlates 
697 Madlon-Kay S, Pesaran B, Daw ND (2013) Action selection in multi-effector decision 698 making. Neuroimage 70:66-79.

699 Mante V, Sussillo D, Shenoy K V, Newsome WT (2013) Context-dependent $700 \quad$ computation by recurrent dynamics in prefrontal cortex. Nature 503:78-84.

701 McGuire LMM, Sabes PN (2009) Sensory transformations and the use of multiple 702 reference frames for reach planning. Nat Neurosci 12:1056-1061.

703 Misslisch H, Tweed D, Hess BJM (2001) Stereopsis Outweighs Gravity in the Control of 704 the Eyes. J Neurosci 21:1-5.

705 Munoz DP, Wurtz RH (1995) Saccade-related activity in monkey superior colliculus I. 706 Characteristics of burst and buildup cells. J Neurophysiol 73:2313-2333.

707 Murdison TS, Leclercq G, Lefèvre P, Blohm G (2015) Computations underlying the 708 visuomotor transformation for smooth pursuit eye movements. J Neurophysiol 709 113:1377-1399.

710 Murdison TS, Paré-Bingley CA, Blohm G (2013) Evidence for a retinal velocity memory 711 underlying the direction of anticipatory smooth pursuit eye movements. J $712 \quad$ Neurophysiol 110:732-747.

713 Newsome WT, Wurtz RH, Komatsu H (1988) Relation of cortical areas MT and MST to 714 pursuit eye movements . II . Differentiation of retinal from extraretinal inputs. J $715 \quad$ Neurophysiol 60:604-620.

716 Palmer J, Huk AC, Shadlen MN (2005) The effect of stimulus strength on the speed and 717 accuracy of a perceptual decision. J Vis 5:376-404.

718 Pozzo T, Berthoz A, Lefort L (1990) Head stabilization during various locomotor tasks in 
humans. I. Normal subjects. Exp Brain Res 82:97-106.

720 Ratcliff R, Rouder JN (2000) A diffusion model account of masking in two-choice letter

721 identification. J Exp Psychol Hum Percept Perform 26:127-140.

722 Riehle A, Requin J (1989) Monkey primary motor and premotor cortex: single-cell 723 activity related to prior information about direction and extent of an intended 724 movement. J Neurophysiol 61:534-549.

725 Roxin A, Ledberg A (2008) Neurobiological models of two-choice decision making can 726 be reduced to a one-dimensional nonlinear diffusion equation. PLoS Comput Biol $727 \quad 4: \mathrm{e} 1000046$.

728 Salinas E, Scerra VE, Hauser CK, Gabriela Costello M, Stanford TR (2014) Decoupling 729 speed and accuracy in an urgent decision-making task reveals multiple 730 contributions to their trade-off. Front Neurosci 8:1-17.

731 Salzman CD, Murasugi CM, Britten KH, Newsome WT (1992) Microstimulation in Visual 732 Area MT: Effects on Direction Discimination Performance. J Neurosci 12:23317332355.

734 Schlicht EJ, Schrater PR (2007) Impact of Coordinate Transformation Uncertainty on 735 Human Sensorimotor Control. J Neurophysiol 97:4203-4214.

736 Schreiber K, Crawford JD, Fetter M, Tweed D (2001) The motor side of depth vision. $737 \quad$ Nature 410:819-822.

738 Shadlen MN, Newsome WT (2001) Neural basis of a perceptual decision in the parietal 739 cortex (area LIP) of the rhesus monkey. J Neurophysiol 86:1916-1936.

740 Shao J, Wei W (1992) A formula for the number of Latin squares. Discrete Math $741 \quad 110: 293-296$. 
742 Siegel M, Buschman TJ, Miller EK (2015) Cortical information flow during flexible

743 sensorimotor decisions. Science 348:1352-1355.

744 Smith PL, Ratcliff R (2004) Psychology and neurobiology of simple decisions. Trends

$745 \quad$ Neurosci 27:161-168.

746 Sober SJ, Sabes PN (2003) Multisensory integration during motor planning. J Neurosci

$747 \quad 23: 6982-6992$.

748 Sober SJ, Sabes PN (2005) Flexible strategies for sensory integration during motor

$749 \quad$ planning. Nat Neurosci 8:490-497.

750 Sommer MA, Wurtz RH (2001) Frontal eye field sends delay activity related to

751 movement, memory, and vision to the superior colliculus. J Neurophysiol 85:1673-

$752 \quad 1685$.

753 Standage D, Blohm G, Dorris MC (2014a) On the neural implementation of the speed-

754 accuracy trade-off. Front Neurosci 8:1-19.

755 Standage D, Wang DH, Blohm G (2014b) Neural dynamics implement a flexible

756 decision bound with a fixed firing rate for choice: A model-based hypothesis. Front

$757 \quad$ Neurosci 8:1-9.

758 Standage D, You H, Wang DH, Dorris MC (2013) Trading Speed and Accuracy by

759 Coding Time: A Coupled-circuit Cortical Model. PLoS Comput Biol 9:e1003021.

760 Thorpe S, Fize D, Marlot C (1996) Speed of processing in the human visual system.

$761 \quad$ Nature 381:520-522.

762 Ungerleider LG, Desimone R (1986) Cortical connections of visual area MT in the 763 macaque. J Comp Neurol 248:190-222.

764 White BJ, Marino RA, Boehnke S, Itti L, Theeuwes J, Munoz DP (2013) Competitive 
765 integration of visual and goal-related signals on neuronal accumulation rate: $A$ 766 correlate of oculomotor capture in the superior colliculus. $\mathrm{J}$ Cogn Neurosci $25: 1754-1768$.

768 Wichmann AF, Hill NJ (2001) The psychometric function: II. Bootstrap-based confidence 769 intervals and sampling. Percept Psychophys 63:1314-1329.

770 Wong YT, Fabiszak MM, Novikov Y, Daw ND, Pesaran B (2016) Coherent neuronal 771 ensembles are rapidly recruited when making a look-reach decision. Nat Neurosci

$772 \quad 19: 1-11$.

773 Yttri EA, Wang C, Liu Y, Snyder LH (2014) The parietal reach region is limb specific and 774 not involved in eye-hand coordination. J Neurophysiol 111:520-532. 


\section{Figure legends}

\section{Figure 1: Task and paradigm.}

778 (A) Participants performed the task under one of eight conditions - four for each

779 response type (saccade or button), organized in a block design. These were

780 combinations of head and/or congruent screen rotations, giving rise to visual motion that

781 was separable across eye, head and shoulder (screen) reference frames. (B) Each trial 782 consisted of a fixation (500 ms), motion (up to $1500 \mathrm{~ms}$ ) and decision epoch.

783 Participants were instructed to determine the direction (left or right) of coherently

784 moving dots randomly chosen at $20 \%, 10 \%$ or $2 \%$ coherence and make their decision 785 using either a horizontal saccade or a button press as quickly and accurately as 786 possible.

788 Figure 2: Rotation condition affected RTs and saccade trajectories.

789 (A) Across coherence levels (columns) specific patterns in RTs across rotational

790 conditions (color-coded, see legend) are shown for participant \#7. Differences in the 791 order of these RT distributions can be seen when comparing saccade (top row) to

792 button responses (bottom row). (B) Compared to control (upper left panel), saccade

793 trajectories were more variable under rotated conditions.

794

795 Figure 3: Psychometric and chronometric functions.

796 Group-level psychometric and chronometric functions revealed that speed and accuracy

797 were not traded-off across rotation conditions, as participants were generally less

798 accurate (psychometric functions, left column) and also slower (chronometric functions, 
799 right column) under rotated conditions. In the chronometric plots, each point represents

800 the group average of the LATER fit parameter $\mu$ approximating the median latency of

801 each condition at each motion strength. Left insets show the point of subjective equality

802 (PSE), which represents the threshold coherence (\%) at which participants chose the

803 correct direction $75 \%$ of the time for the 2AFC task. On the same scale but with different

804 units (\% coherence per \% correct), right insets also show the just-noticeable difference

805 (JND), which approximates the amount of variability in the psychometric function using

806 the inverse of the slope around the $75 \%$ PSE, scaled for 2AFC tasks. Each of these

807 insets reveals a consistent trend in the median and variability of performance across

808 rotation conditions.

809

810 Figure 4: Variability of rotational effects on performance across participants.

811 Changes in latency (top row), percent error (middle row) and reward rate (bottom row)

812 across coherence level (columns), with left axes representing scale for single participant

813 changes (colored line segments, see legend for participant numbers) and right axes

814 representing group-level average changes across rotation conditions (color-coded

815 bars). Each vertex of the line segments represents one rotation condition, in line with

816 the colored bars at the bottom. Note that for direct comparisons with latency and

817 percent error changes we inverted the vertical axes for reward rate changes.

819 Figure 5: Reference frame predictions and analysis.

820 (A) Effector specific reference frame prediction matrices. Each cell represents a specific

821 reference frame and the predicted effect size for the corresponding rotation condition. 
822 For example, if motion evidence were coded according to an eye-centered reference

823 frame, for the condition in which only the motion stimulus were rotated (condition S) we

824 would expect a large (black shading) reference frame transformation-induced stochastic

825 effect on the coded evidence signal in both saccade and button response conditions.

826 (B) Participant R-squared coefficients for correlation analysis between prediction

827 matrices in panel (A) and observed changes in latency (top row), percent error (middle

828 row), and reward rate (bottom row), across coherence levels (columns). Participant

829 color code is the same as in previous figures, and black symbols represent across-

830 participant means. Open circles and filled squares represent R-squared coefficients for

831 saccade responses and for button responses, respectively. Pure eye- (red), head-

832 (blue), and shoulder-centered (green) reference frame predictions are represented with

833 large filled circles.

834

835 Figure 6: Control experiment conditions and example RT distributions.

836 (A) Representation of added rotational conditions in which the speed of the motion

837 stimulus was increased to compensate for the loss of horizontal motion in the $S$

838 condition (S-spd, left) and in which the saccadic responses were also rotated to match

839 the rotated motion axis, thus isolating the effect of head roll in the transformation (HR-S-

$840 \mathrm{RR}$, right). (B) Exemplar participant RT distributions for saccades (top row) and button

841 responses (bottom row), showing control (black), S (cyan), S-spd (green) and HR-S-RR

842 (purple) conditions across coherence levels (columns). 

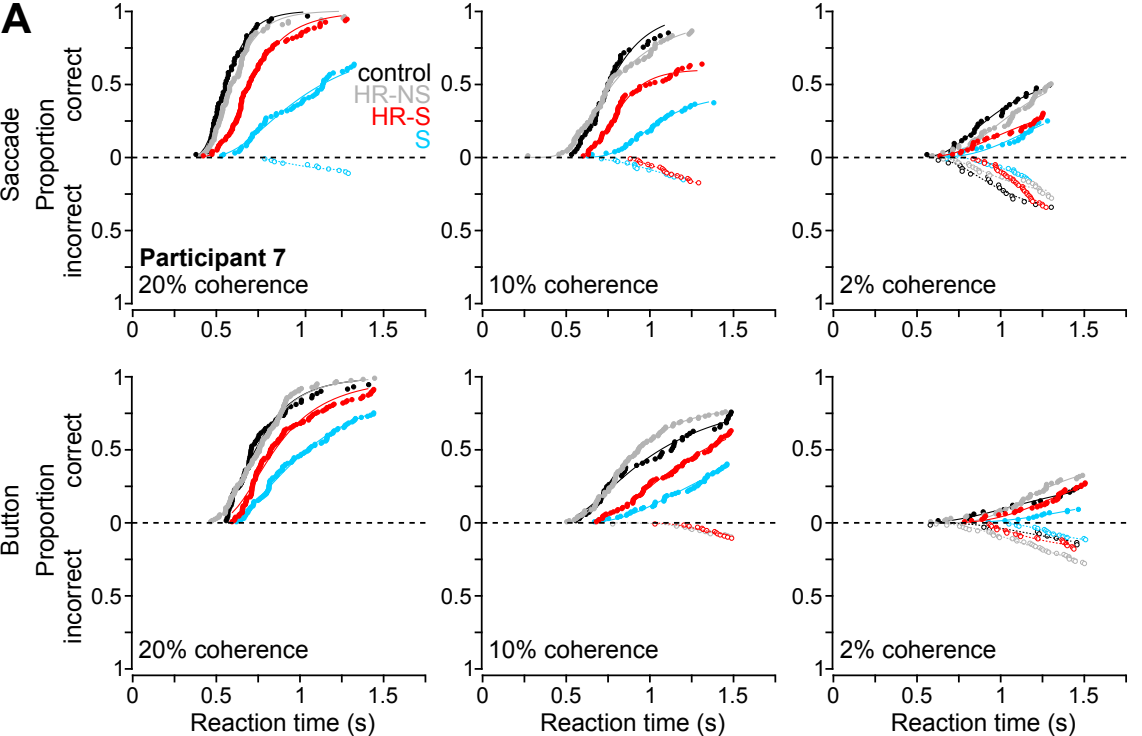

Reaction time (s)

Reaction time (s)

Reaction time (s)

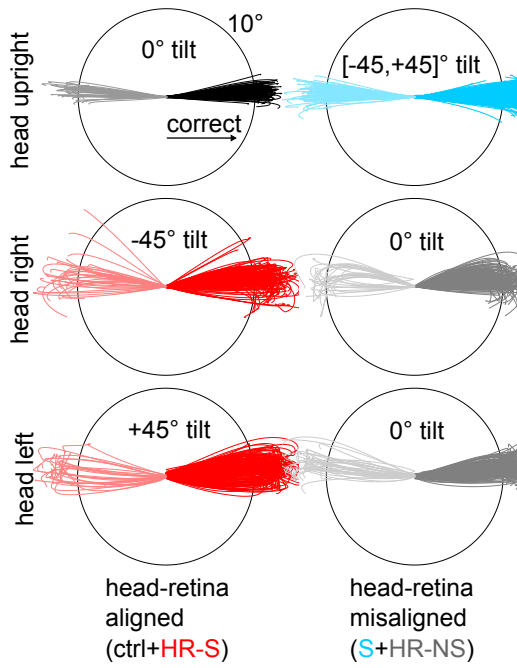




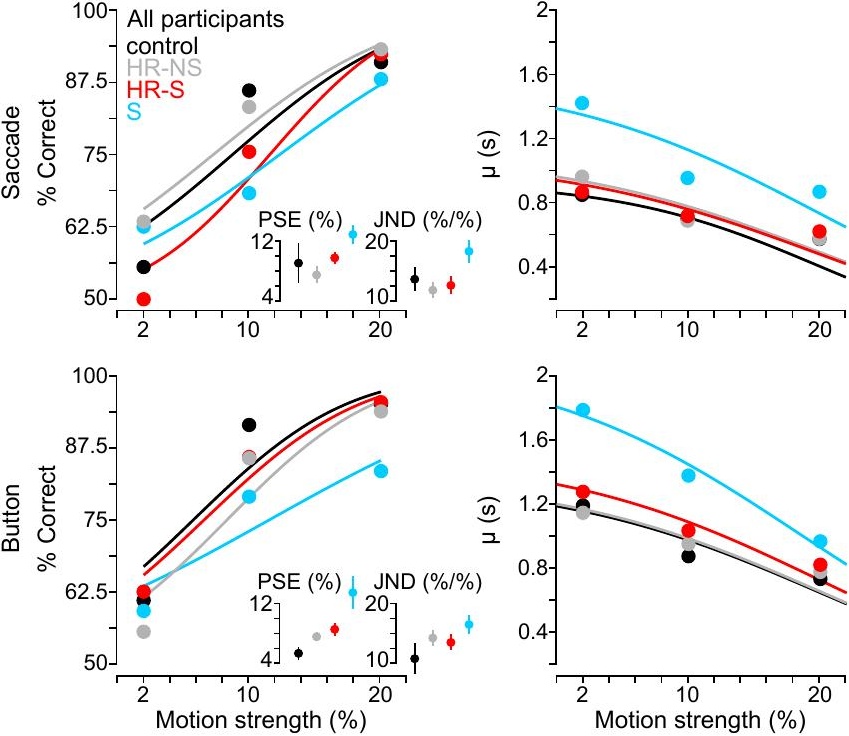



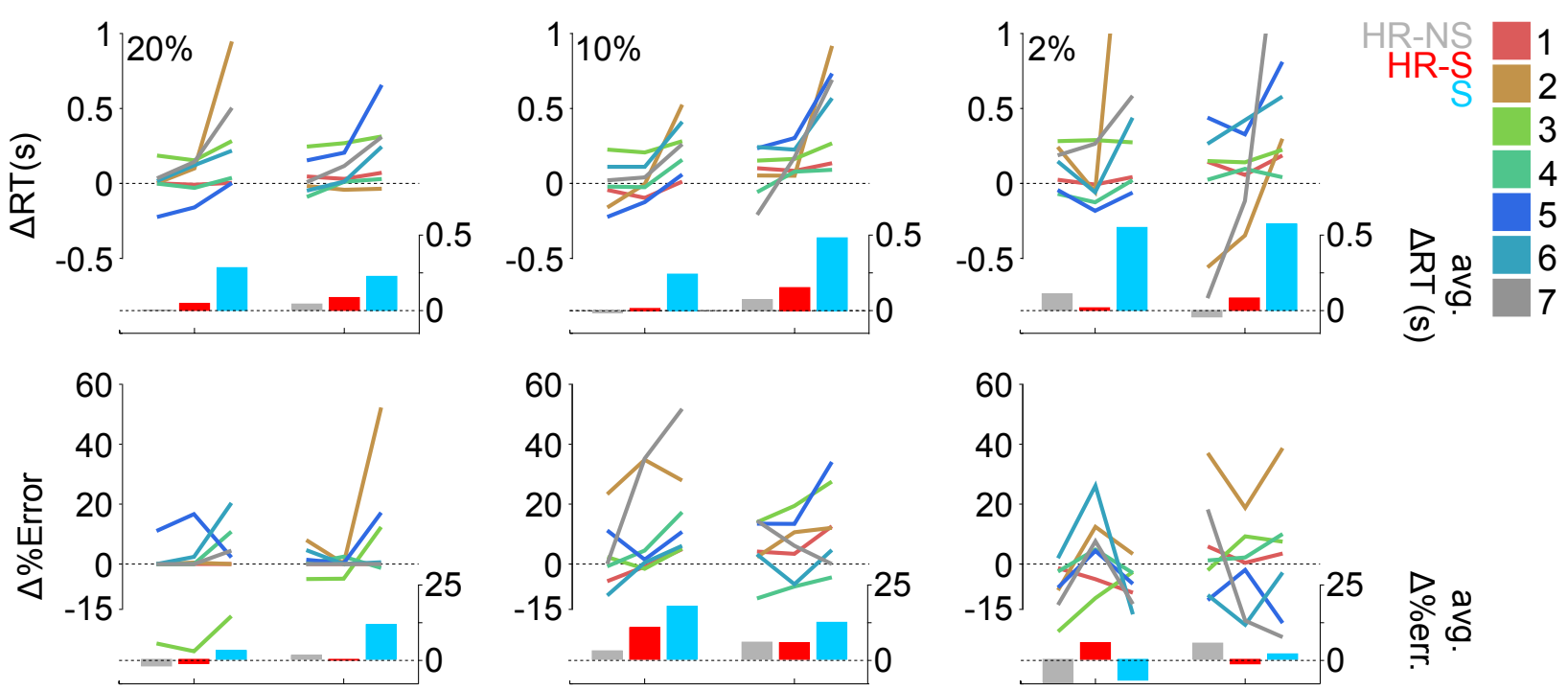

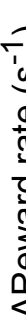

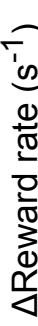

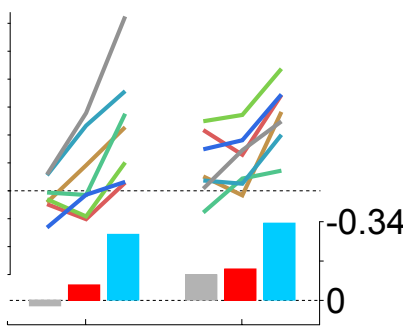

Saccade Button
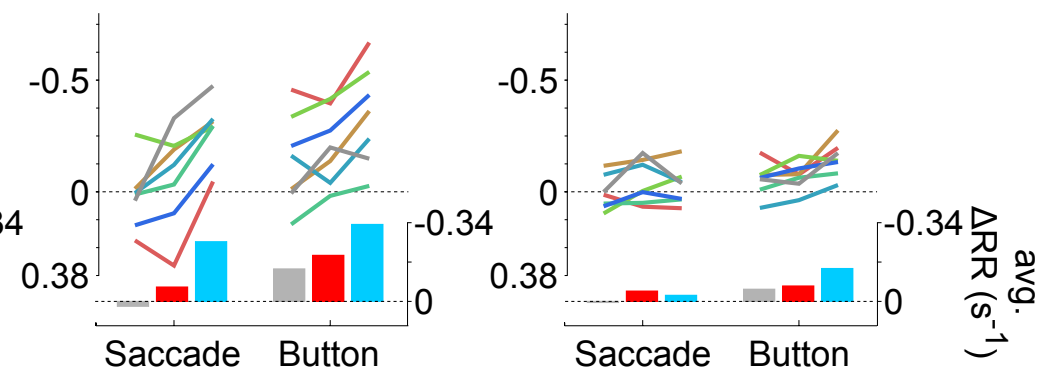


\section{Rotation condition}

HR-NS HR-S

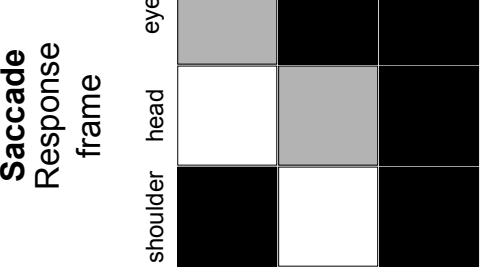

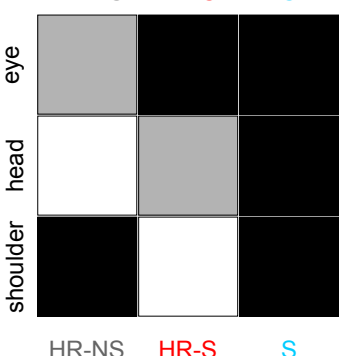

동

일 을

ต

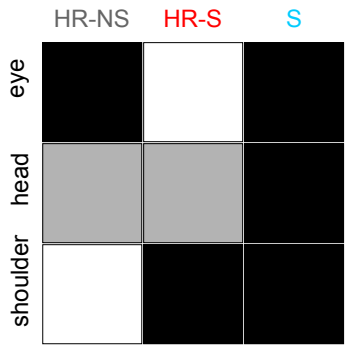

expected

effect size

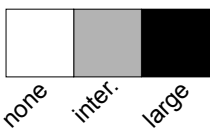

B
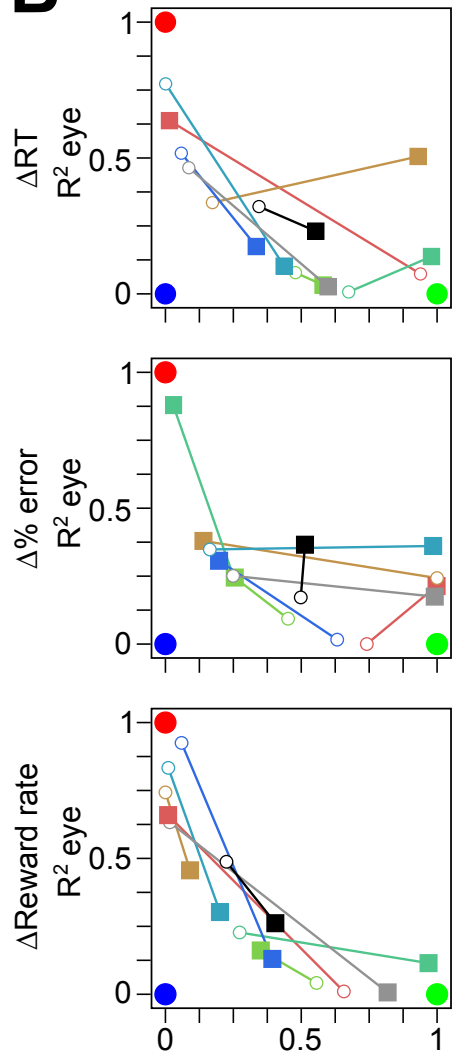

$R^{2}$ shoulder
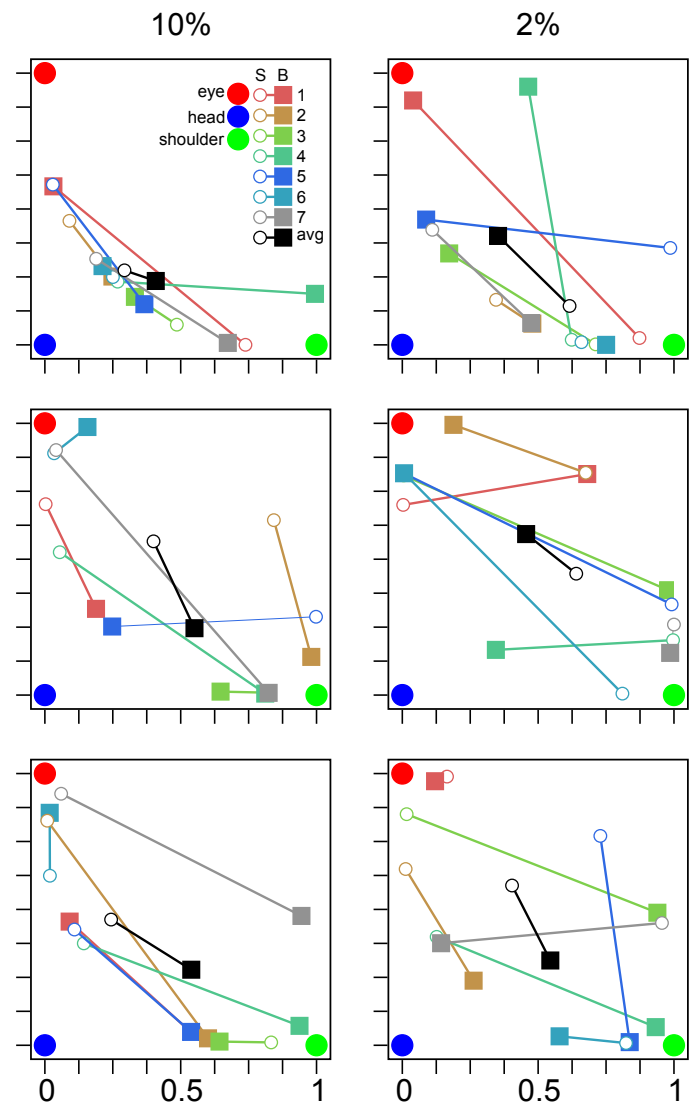

$\mathrm{R}^{2}$ shoulder

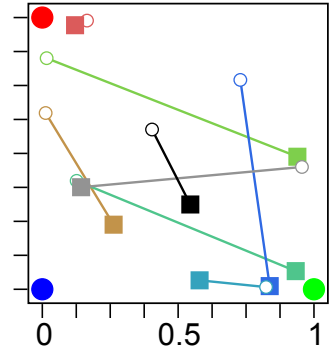

$\mathrm{R}^{2}$ shoulder 
